\title{
Model Structures and Algorithms for Identification of Aerodynamic Models for Flight Dynamics Applications
}

\author{
Ravi K. Prasanth, Vladislav Klein ${ }^{\dagger}$ Patrick C. Murphy ${ }^{\ddagger}$ and Raman K. Mehra ${ }^{\S}$
}

\begin{abstract}
This paper describes model structures and parameter estimation algorithms suitable for the identification of unsteady aerodynamic models from input-output data. The model structures presented are state space models and include linear timeinvariant (LTI) models and linear parameter-varying (LPV) models. They cover a wide range of local and parameter dependent identification problems arising in unsteady aerodynamics and nonlinear flight dynamics. We present a residue algorithm for estimating model parameters from data. The algorithm can incorporate apriori information and is described in detail. The algorithms are evaluated on the F-16XL wind-tunnel test data from NASA Langley Research Center. Results of numerical evaluation are presented. The paper concludes with a discussion major issues and directions for future work.
\end{abstract}

\section{Introduction}

A model is a useful representation of system dynamics. The precise meaning of "useful" is given by the application for which the model is intended. As a general rule, useful models of engineering systems must be computationally efficient. Another important requirement is the ability to accurately describe system behaviors under different conditions, i.e., predictive power of the model. These two requirements are competing interests in that models that predict many system behaviors tend to be computationally hard and vice versa. For instance, Navier-Stokes equations have a lot more predictive power than the aerodynamic models discussed in this paper, but they are also numerically much harder. The modeler's task is then to develop models that balance computational and predictive requirements perhaps by neglecting fine scale structure and considering only those features that are dominant in the application. This is, of course, easier said than done in most flight dynamics problems where distributed phenomena interact to produce complex dynamical behaviors. A good example is tail-buffeting at high angles of attack in which vortices shed

${ }^{*}$ Senior Technical Fellow, Scientific Systems Company, Woburn, MA. AIAA Member

${ }^{\dagger}$ Professor, George Washington University, Washington, D.C. AIAA Member

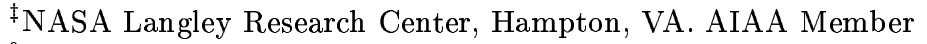

${ }^{\S}$ President, Scientific Systems Company, Woburn, MA. AIAA Member

1 of 18

American Institute of Aeronautics and Astronautics 
from wing impinge tail surface causing structural vibrations and eventually fatigue. Identification of models from experimental data may be the only avenue in such problems. In other problems where first principles can be used to derive models such as in rigid body dynamics, identification of model parameters such as stability derivatives from experimental data is required. Researchers have also developed simple models for complicated but special flow problems by considering macroscale flow physics. Such models also contain parameters that must be estimated from experimental data. ${ }^{5,6,8,10}$

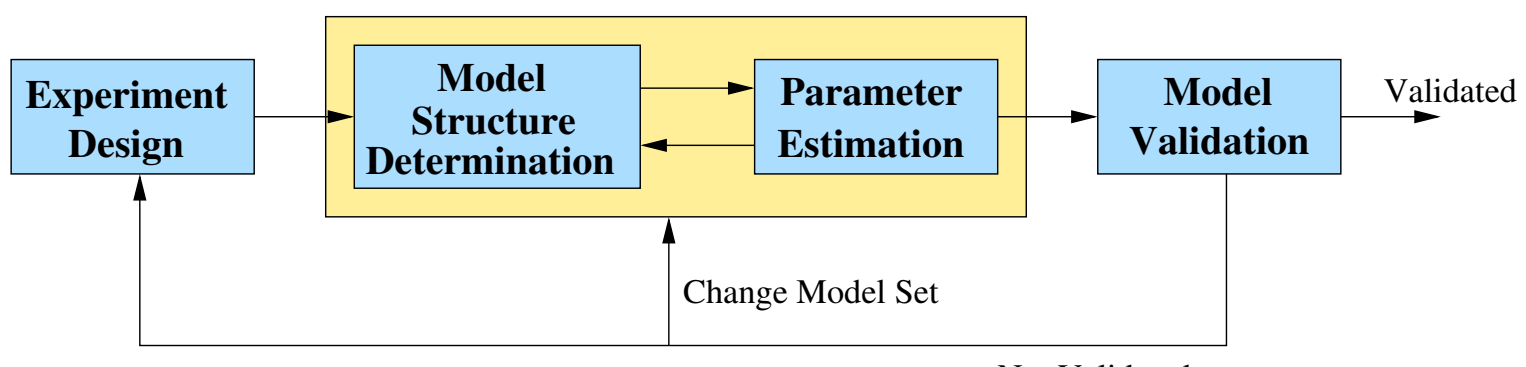

Not Validated

Figure 1. Steps in system identification

System identification is an iterative process consisting of experiment design, model structure determination, parameter estimation and model validation (Figure 1). ${ }^{7,13}$ Experiment design is concerned with the selection of test inputs and measurement variables, their sampling and conditioning, and other aspects that are directly related to the notion of an informative experiment. An experiment is said to be informative with respect to a model set if the experimental data allows discrimination between models in the set. Model structure selection and parameter estimation are simultaneous tasks, though in practice, they are performed sequentially with model structure being selected first. The structure of a model is defined in terms of its nature (eg. linear black-box, Wiener, neural-network), order (number of free variables) and parameterization (e.g state space in modal coordinates). There are several considerations that go into model structure selection including apriori knowledge, flexibility and algorithmic complexity. After selecting a model structure, parameters of the model are estimated from experimental data. Minimum mean square estimation (MMSE) and maximum likelihood estimation $(\mathrm{MLE})^{7,13,15}$ are the most common methods of parameter estimation. Numerical procedures for estimation range from least squares to the expectation-maximization (EM) algorithm. ${ }^{4}$ The final step of model validation is to determine the "goodness" of an identified model, i.e, the extent to which it is useful in its intended application. Thus, a model identified for controller design may be deemed valid if the resulting controller produces satisfactory performance. When model validation fails, it may be due to the choice of model structure and the lack of information in experimental data. As shown in Figure 1, the steps are iterated until a validated model is found. Details of these steps and the iterative process can be found in Ref.13, 14, 15 .

This paper ${ }^{\mathrm{a}}$ describes time domain identification methods suitable for the modeling of unsteady and nonlinear aerodynamics behaviors arising in flight dynamics. Section II begins with model

\footnotetext{
${ }^{\mathrm{a}} \mathrm{A}$ detailed version of the paper can be obtained from the authors
} 
structures for modeling dynamics in the neighborhood of an operating condition, for example, fixed Mach number or fixed dynamic pressure. Such models are local models with a limited region of validity. The section also presents parameter-dependent model structures for modeling dynamics over a range of parameter values. We refer to such models as global models though they are not suitable models for certain global behaviors. The model structures are illustrated with examples relating them to well-known models such as the indicial response function models. A residue based algorithm for parameter estimation is given in Section III. The algorithms are evaluated on the F-16XL wind-tunnel test data from NASA Langley Research Center. Results of numerical evaluation are presented in Section IV. Conclusions and recommendations for future work are given in Section V.

System identification methods have been used widely in aircraft applications. The special issue of the AIAA Journal of Aircraft ${ }^{1}$ and the invited session at the AIAA Atmospheric Flight Mechanics ${ }^{2}$ contain papers describing many techniques and a broad range of recent applications. The main contributions of this paper are a new algorithm for identifying state space models of unsteady aerodynamic effects and its limited validation using F-16XL wind-tunnel test data.

\section{Local and Global Parameter Dependent Model Structures}

A model structure specifies how test inputs and external noises affect observed outputs. We only describe the relationship between test inputs and observed outputs to keep the presentation simple.

\section{A. Unstructured Linear Time-Invariant (LTI) Models}

Consider a continuous-time LTI state space model:

$$
\begin{aligned}
& \dot{x}=A x+B u \\
& y=C x+D u
\end{aligned}
$$

where $A \in \mathbb{R}^{n \times n}, B \in \mathbb{R}^{n \times m}, C \in \mathbb{R}^{l \times n}$, and $D \in \mathbb{R}^{l \times m}$. This model is completely determined by an order parameter $n$ and the state space matrices $(A, B, C, D)$. It is an unstructured model because the state space matrices have no specific structure and are allowed to take on any value. When the order parameter $n$ is fixed, we get a fixed order unstructured model.

\section{B. Structured LTI Models}

In many applications, the natural frequencies and damping coefficients of system modes are known apriori with some confidence, e.g, $\omega_{n} \pm 0.2 \omega_{n}$ for a $20 \%$ variation from the nominal natural frequency $\omega_{n}$. Physical reasoning may suggest the use of first order exponentially decaying or the second order decaying oscillatory model ${ }^{5,6,8,10}$ or a combination of both. Thus, the $A$ matrix takes the form:

$$
A=\operatorname{diagonal}\left\{r_{1}, \cdots, r_{N_{r}},\left[\begin{array}{cc}
0 & 1 \\
-\omega_{1}^{2} & -2 \zeta_{1} \omega_{1}
\end{array}\right], \cdots,\left[\begin{array}{cc}
0 & 1 \\
-\omega_{N_{c}}^{2} & -2 \zeta_{N_{c}} \omega_{N_{c}}
\end{array}\right]\right\}
$$

3 of 18

American Institute of Aeronautics and Astronautics 
where $\left\{r_{k}\right\}_{k=1}^{N_{r}}$ are the real eigenvalues and $\left\{\left(\omega_{k}, \zeta_{k}\right)\right\}_{k=1}^{N_{c}}$ are the eigenvalues with non-zero imaginary parts written in terms of natural frequency $\omega_{k}$ and damping coefficient $\zeta_{k}$.

The model given by (1) and (2) is specified by the order parameters $\left(N_{r}, N_{c}\right)$, the eigenvalue locations $\left\{r_{k}\right\}_{k=1}^{N_{r}}$ and $\left\{\left(\omega_{k}, \zeta_{k}\right)\right\}_{k=1}^{N_{c}}$, and the matrices $(B, C, D)$. If the postulated model (1) and (2) is used for system identification, some or all parameters in $A$ may be known apriori with a given level of confidence. Then, apriori information on the parameters is described by a set $\Delta$ of admissible eigenvalue locations:

$$
\Delta=\left\{\left(\left\{r_{k}\right\}_{k=1}^{N_{r}},\left\{\left(\omega_{k}, \zeta_{k}\right)\right\}_{k=1}^{N_{c}}\right): r_{j}^{\min } \leq r_{j} \leq r_{j}^{\max }, \omega_{k}^{\min } \leq \omega_{k} \leq \omega_{k}^{\max }, \cdots\right\}
$$

where the bounds specify confidence intervals for each eigenvalue.

Example II.1 (Structured model) Consider an indicial response function model:

$$
\begin{aligned}
y(t) & =c_{1} \int_{0}^{t} e^{a_{1}(t-\tau)} \dot{\alpha}(\tau) d \tau+c_{2} \int_{0}^{t} e^{a_{2}(t-\tau)} \ddot{\alpha}(\tau) d \tau \\
& =\int_{0}^{t} G(t-\tau)\left[\begin{array}{c}
\dot{\alpha} \\
\ddot{\alpha}
\end{array}\right] d \tau
\end{aligned}
$$

where $a_{1}<0, a_{2}<0, c_{1}$ and $c_{2}$ are unknown real numbers. This dynamical system in more general form appears in the work of Klein and Murphy ${ }^{10}$ where it models the unsteady contribution to aerodynamic coefficients in one degree of freedom motion. The strict negativity of $a_{1}$ and $a_{2}$ guarantee that bounded test inputs ( $\dot{\alpha}$ and $\ddot{\alpha}$ ) produce bounded outputs $(y)$. The integral representation can be converted to state space form as:

$$
\begin{aligned}
& \dot{x}=\left[\begin{array}{cc}
a_{1} & 0 \\
0 & a_{2}
\end{array}\right] x+\left[\begin{array}{ll}
1 & 0 \\
0 & 1
\end{array}\right]\left[\begin{array}{c}
\dot{\alpha} \\
\ddot{\alpha}
\end{array}\right] \\
& y=\left[\begin{array}{ll}
c_{1} & c_{2}
\end{array}\right] x
\end{aligned}
$$

$A$ relationship between model equations (6) and aircraft equations of motion will be shown in Section IV. In (6a), the A-matrix has a specific structure. Let us assume that $a_{1}$ satisfies:

$$
a_{\min } \leq a_{1} \leq a_{\max }<0
$$

and that no information is available on the remaining parameters (other than strict negativity of $\left.a_{2}\right)$. Then, $\Delta$ takes the form:

$$
\Delta=\left\{\left(a_{1}, a_{2}\right): a_{\min } \leq a_{1} \leq a_{\max }<0, a_{2}<0\right\}
$$

Thus, indicial response function models of the form (4) are structured LTI models. We make two important observations. First, the number of (linearly independent) terms in the convolution form is equal to the model order parameter (state space dimension). Second, the B-matrix is a constant. This is true of all LTI models. 


\section{Linear Parameter Varying (LPV) Global Models}

Many aerodynamic and flight dynamics behaviors result from changes in system dynamics brought about by changes in physical parameters. The LTI models described in the previous section are not appropriate for such systems. We present generalizations of the local models of the previous sections that can capture parameter-dependent phenomena in this section.

Consider the system:

$$
\begin{aligned}
& \dot{x}=A(\alpha) x+B(\alpha) u \\
& y=C(\alpha) x+D(\alpha) u
\end{aligned}
$$

where $A(\alpha) \in \mathbb{R}^{n \times n}, B(\alpha) \in \mathbb{R}^{n \times m}, C(\alpha) \in \mathbb{R}^{1 \times n}$, and $D(\alpha) \in \mathbb{R}^{1 \times m}$. A system of this form is called a linear parameter-varying $(L P V)$ system. The system matrices are functions of angle of attack $\alpha$ and, during system operation, the angle of attack changes with time. Thus, along each possible $\alpha$ trajectory, (7) is a linear time-varying system. Since the specific trajectory taken by $\alpha$ can change from one run to another, the above system is not a single linear time-varying system, but a collection of linear time-varying systems. It is a global model in this restricted sense.

Example II.2 (Indicial function vs. LPV model) The integral kernel in Example II.1 has the general form:

$$
G(t-\tau ; \alpha(\tau))=\sum_{k} G_{k}(t-\tau) \alpha(\tau)^{k}
$$

which implies that the dynamical part of those models, given by $G_{k}$ 's, is LTI. Consider the state space model:

$$
\begin{aligned}
& \dot{x}=a(\alpha) x+\dot{\alpha} \\
& y=c(\alpha) x
\end{aligned}
$$

where $a$ and $c$ are scalar polynomial functions of the parameter $\alpha$. When $\alpha$ is a specified function of time, the system equations constitute a linear time-varying system. We have (for zero initial condition):

$$
y(t)=c(\alpha(t)) \int_{0}^{t} e^{\int_{\tau}^{t} a(\alpha(s)) d s} \dot{\alpha}(\tau) d \tau
$$

whose kernel is:

$$
e^{\int_{\tau}^{t} a(\alpha(s)) d s}
$$

which cannot be written as in Example II.1. Since the state space matrices are functions of $\alpha$, we can describe $\alpha$-dependent phenomenon with this model. In Section IV, the model given by equations (8) is used for aircraft parameter estimation. 


\section{Algorithms for Model Parameter Estimation}

A number of techniques that go under the name of subspace algorithms ${ }^{16,23}$ have been developed for unstructured LTI model parameter estimation. In this section, we describe residue-based algorithms for model parameter estimation of structured LTI and LPV models. These algorithms have finite data guarantees for noise-free data and asymptotic guarantees for noisy data even in the multi-input multi-output (MIMO) case provided that the system that generated the data is in the model set.

Let $\left\{u_{k}\right\}_{k=1}^{N}$ denote the applied input and $\left\{y_{k}\right\}_{k=1}^{N}$ denote the measured output. Fix a model structure. A model is then defined by a model parameter vector that belongs to some set $\mathcal{P}$. Choose a model by fixing model parameter values to $p \in \mathcal{P}$. Let us denote by:

$$
\left\{y_{1}^{p}, y_{2}^{p}, \cdots, y_{N}^{p}\right\}
$$

the output obtained by driving the chosen model with the input $\left\{u_{1}, \cdots, u_{N}\right\}$. The parameter estimation problem is to find a model parameter that minimizes:

$$
\frac{1}{N} \sum_{k=1}^{N}\left(y_{k}-y_{k}^{p}\right)^{\prime}\left(y_{k}-y_{k}^{p}\right)
$$

This is not a standard least squares problem because some of the parameters (e.g. model order) may be discrete-valued and, more generally, constrained. To reduce the computational requirements, the model order (and all other discrete-valued parameters) are fixed and the best fixed-order model is calculated. The model order and other discrete-valued parameters are then selected using information criteria such as those of Akaike (AIC), Bayes (BIC) and Rissanen (MDL) by performing a sequence of fixed order model identifications. Our focus in this section is on fixed order model identification.

Consider the fixed order structured LTI model given by equations (1) and (2). The model parameters are the parameters in the state space matrices $A, B, C$ and $D$. The parameter estimation problem can be written as:

$$
\min _{p \in \mathcal{P}} \frac{1}{N} \sum_{k=1}^{N}\left(y_{k}-y_{k}^{p}\right)^{\prime}\left(y_{k}-y_{k}^{p}\right)=\min _{\left(\left\{r_{k}\right\},\left\{\omega_{i}, \zeta_{i}\right\}\right) \in \Delta} \min _{B, C, D} \frac{1}{N} \sum_{k=1}^{N}\left(y_{k}-y_{k}^{p}\right)^{\prime}\left(y_{k}-y_{k}^{p}\right)
$$

where $\Delta$ specifies the apriori information on the parameters in $A$. We will now show that the inner-most minimization problem over the matrices $B, C$ and $D$ is a linear least squares problem.

Suppose that the pole locations are fixed as in the inner minimization problem. Then, due to its modal structure, the $A$-matrix is fixed by its definition in (2). Choose any $B, C$ and $D$. Then, we have:

$$
\begin{aligned}
y(s) & =G(s) u(s)=\left(D+C(s I-A)^{-1} B\right) u(s) \\
& =\left(D+R \widehat{C}(s I-\widehat{A})^{-1} \widehat{B}\right) u(s)
\end{aligned}
$$

6 of 18

American Institute of Aeronautics and Astronautics 


$$
\begin{aligned}
& =\left[\begin{array}{ll}
D & R
\end{array}\right]\left[\widehat{C}(s I-\widehat{A})^{-1} \widehat{B}\right] u(s) \\
& =\left[\begin{array}{ll}
D & R
\end{array}\right] \widehat{T}(s) u(s)
\end{aligned}
$$

where $\widehat{A}, \widehat{B}$ and $\widehat{C}$ are known matrices independent of $B, C$ and $D, R$ is the block-row matrix of residues that depends on $B$ and $C$, and the definition of the intermediate transfer function $\widehat{T}$ is clear from (10). Note that $\widehat{T}$ is a known transfer function. Figure 2 shows the relationship in equation (10) using block diagrams.

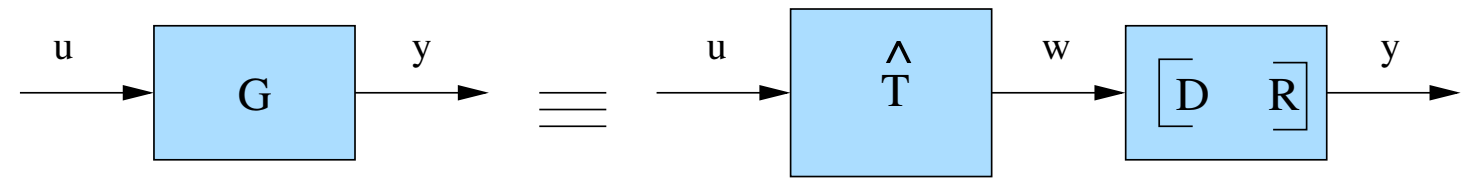

Figure 2. An equivalent way of generating output from input

The main observation is that the intermediate signal $w$ defined as:

$$
w(s)=\widehat{T}(s) u(s)
$$

(see Figure 2) is known since both the applied control input $u$ and the intermediate transfer function $\widehat{T}$ are known. Thus, the inner minimization problem can be restated as:

$$
\min _{B, C, D} \frac{1}{N} \sum_{k=1}^{N}\left(y_{k}-y_{k}^{p}\right)^{\prime}\left(y_{k}-y_{k}^{p}\right)=\min _{D, R} \frac{1}{N} \sum_{k=1}^{N}\left(y_{k}-\left[\begin{array}{ll}
D & R
\end{array}\right] w_{k}\right)^{\prime}\left(y_{k}-\left[\begin{array}{ll}
D & R
\end{array}\right] w_{k}\right)
$$

which shows that it is a standard linear least squares problem. A step by step procedure is given below.

Algorithm III.1 (Residue Algorithm) Let $\left(\left\{r_{k}\right\},\left\{\omega_{i}, \zeta_{i}\right\}\right) \in \Delta$ be the given pole locations and $A$ be the associated modal matrix as in (2).

Step 1: Form the matrices $\widehat{A}, \widehat{B}$ and $\widehat{C}$.

Step 2: Obtain the intermediate signal $w$ by calculating the response of the intermediate transfer function $\widehat{T}$ to the input $u$. This can be done by forming a state space model for $\widehat{T}$ using $\widehat{A}, \widehat{B}$ and $\widehat{C}$, and integrating the state equations.

Step 3: Solve the least squares problem (11) to get $D$ and $R$.

Step 4: Reduce $(\widehat{A}, \widehat{B}, R \widehat{C}, D)$ to get a state space model that best approximates the data.

Thus, an algorithm to determine the unknown parameters of a structured LTI model is the following:

7 of 18

American Institute of Aeronautics and Astronautics 
Algorithm III.2 (Structured Identification Algorithm) The following steps identify a structured LTI state space model given by equations (1) and (2) from input-output data.

Step 1: Initialize the parameters of $A$ matrix (pole locations $\left(\left\{r_{i}\right\},\left\{\omega_{i}, \zeta_{i}\right\}\right)$ )

Step 2: Loop until convergence

(a) Apply residue algorithm to solve the inner minimization problem. Determine the least squares approximation error from Step 3 of the residue algorithm.

(b) Calculate (numerical) derivative of least squares approximation error with respect to pole locations.

(c) Update pole locations using Newton's method and return to step (a).

Step 3: Upon convergence, form state space model using the residue algorithm.

For the LPV model given by (7), a similar algorithm can be given provided that the $A$-matrix is diagonal:

$$
A(\alpha)=\text { diagonal }\left\{r_{1}(\alpha), \cdots, r_{n}(\alpha)\right\}
$$

In this case, algebraic manipulations similar to those used in the derivation of residue algorithm show that the LPV model (7) is equivalent to a model of the form:

$$
\begin{aligned}
& \dot{\hat{x}}=A(\alpha) \hat{x}+\widehat{B} u \\
& y=\widehat{C}(\alpha) \hat{x}+D(\alpha) u
\end{aligned}
$$

where $\widehat{B}$ is a known constant matrix. As before, by moving the unknown parameters in the $B$ matrix of the original LPV model (7) into $C$ matrix of the modified LPV model (13), the overall identification problem can be cast as an outer minimization problem over the parameters in $A$ and an inner least squares minimization problem over the parameters in $\widehat{C}$ and $D$. Thus, we have the following procedure for LPV model identification.

Algorithm III.3 (LPV Model Identification Algorithm) The following steps identify a LPV state space model given by equations (7) and (12) from input-output data.

Step 1: Initialize parameters of A matrix

Step 2: Loop until convergence

(a) Construct the modified LPV model (13) and simulate equation (13a) to get state time histories.

(b) Formulate and solve least square minimization problem to get the parameters in $\widehat{C}$ and $D$.

8 of 18

American Institute of Aeronautics and Astronautics 
(c) Determine the least squares approximation error.

(d) Calculate (numerical) derivative of least squares approximation error with respect to parameters of A matrix.

(c) Update parameters of A matrix using Newton's method and return to step (a).

Step 3: Upon convergence, form LPV state space model from steps 2a-2b.

\section{F-16XL Data Analysis}

This section presents an overview of the F-16XL data and the identification of unsteady aerodynamic models from the data using the methods described earlier.

\section{A. F-16XL Wind-Tunnel Data}

The F-16XL wind tunnel test data used in this study was collected from static and dynamic tests performed as part of other work $^{9,10,12}$ using the NASA Langley 12-Foot Low-Speed Wind Tunnel. A sketch of the 10\%-scale F-16XL model is shown in Figure 3. For the tests, the model was mounted on a dynamic hydraulically actuated test rig through a six-component strain gauge balance. The mounting was capable of rotating the model through an angle of attack range of $-5^{\circ}$ to $80^{\circ}$ about the center of gravity location at $0.558 \mathrm{c}$. The maximum capability of the dynamic test rig was $260 \mathrm{deg} / \mathrm{sec}$ in pitch rate and $2290 \mathrm{deg} / \mathrm{sec}^{2}$ in pitch acceleration. The tests were conducted at a dynamic pressure of 4 psf and a Reynolds number of $10^{6}$ based on the mean aerodynamic chord. The data were collected with the leading-edge flaps at $0^{\circ}$ deflection. Data were sampled at $100 \mathrm{~Hz}$ and subsequently filtered using a $6 \mathrm{~Hz}$ low pass filter.

The test data set consists of time histories of the lift coefficient $C_{L}$ and the pitching moment coefficient $C_{M}$ from static experiments and dynamic experiments with small amplitude oscillatory, large amplitude oscillatory and ramp test inputs. Static data were obtained for angles of attack from $-5^{\circ}$ to $80^{\circ}$ at zero side-slip and zero trailing-edge surface (flaperons and elevons) deflection. The angle of attack $(\alpha)$ test input used to collect the oscillatory data has the form:

$$
\alpha(t)=\alpha_{0}+\alpha_{A} \sin (2 \pi f t)
$$

where $\alpha_{0}$ is the mean angle of attack, $\alpha_{A}$ is the amplitude and $f$ is the frequency in $\mathrm{Hz}$.

For small amplitude experiments,

$$
\alpha_{A}=5^{\circ}, 20.8^{\circ} \leq \alpha_{0} \leq 61.1^{\circ} \text { and } f=0.6,1.0,1.41,1.75 \text { and } 2.94
$$

Thus, each data file corresponds to an experimental condition indexed by $\left(\alpha_{0}, f\right)$ and contains information related to system behavior in the neighborhood of $\left(\alpha_{0}, f\right)$. The large amplitude data

9 of 18

American Institute of Aeronautics and Astronautics 


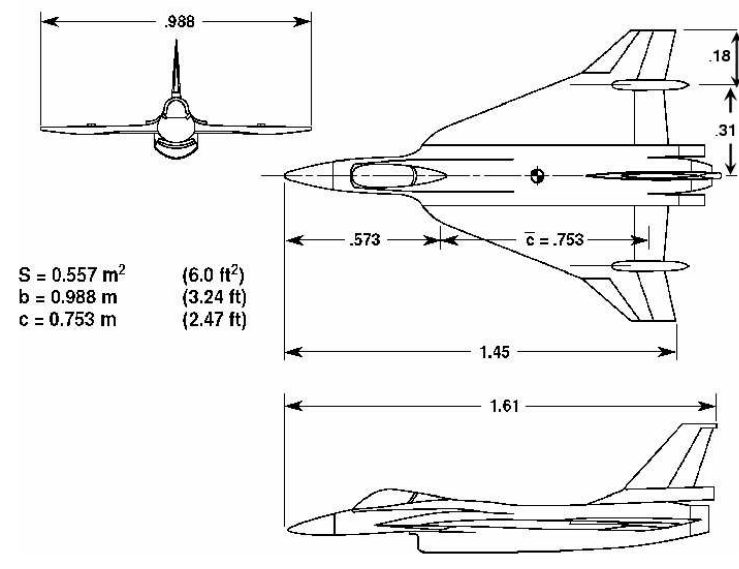

(a) F-16XL $10 \%$ scale model dimensions

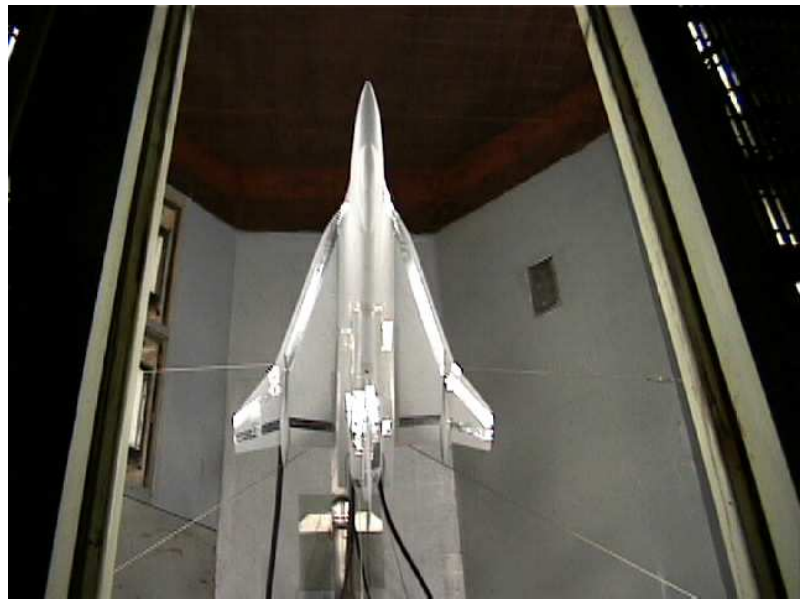

(b) F-16XL $10 \%$ model in wind tunnel

Figure 3. F-16XL scaled model used for data collection

set contains 18 data files of $C_{L}$ and $C_{M}$ with

$$
\alpha_{0}=35^{\circ}, \alpha_{A}=10^{\circ}, 20^{\circ} \text { and } 35^{\circ} \text { and } f=0.083,0.25,0.42,0.58,0.75 \text { and } 1
$$

The ramp data consist of several input-output pairs, the input being angle of attack $\alpha$ and the output being $C_{L}$ and $C_{M}$. Each pair is from a different experiment in which $\alpha$ was varied as:

$$
\alpha(t)=\alpha_{0}+\dot{\alpha} t
$$

where

\begin{tabular}{|c|l|l|}
\hline \hline Record & $\dot{\alpha}(\mathrm{deg} / \mathrm{sec})$ & $\alpha_{0}(\mathrm{deg})$ \\
\hline Record 1-9 (ramp up) & $18,55,92,128,165,220,1,5,10$ & 0 \\
\hline Record 10-15 (ramp down) & $-18,-55,-92,-128,-165,-220$ & 70 \\
\hline \hline
\end{tabular}

Figure 4 presents plots of $C_{L}(\alpha(t))$ from small amplitude oscillatory test data set at two frequencies and three values of $\alpha_{0}$. At $\alpha_{0}=20^{\circ}$, the phase difference between $\alpha$ and $C_{L}$ is essentially constant for both frequencies. At higher $\alpha_{0}$, however, the variation of $C_{L}$ with $\alpha$ changes with $\alpha_{0}$ and frequency. This behavior can be explained by an indicial response function model whose parameters depend on $\alpha$ and $\dot{\alpha}$. Then, the model can be reformulated to the state-variable form given by equations (6). 


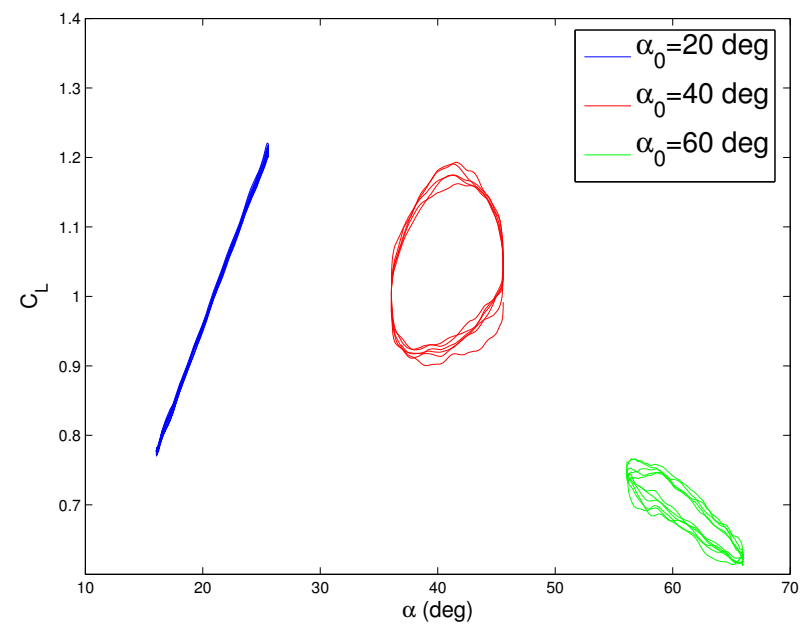

(a) $f=0.6 \mathrm{rad} / \mathrm{s}$

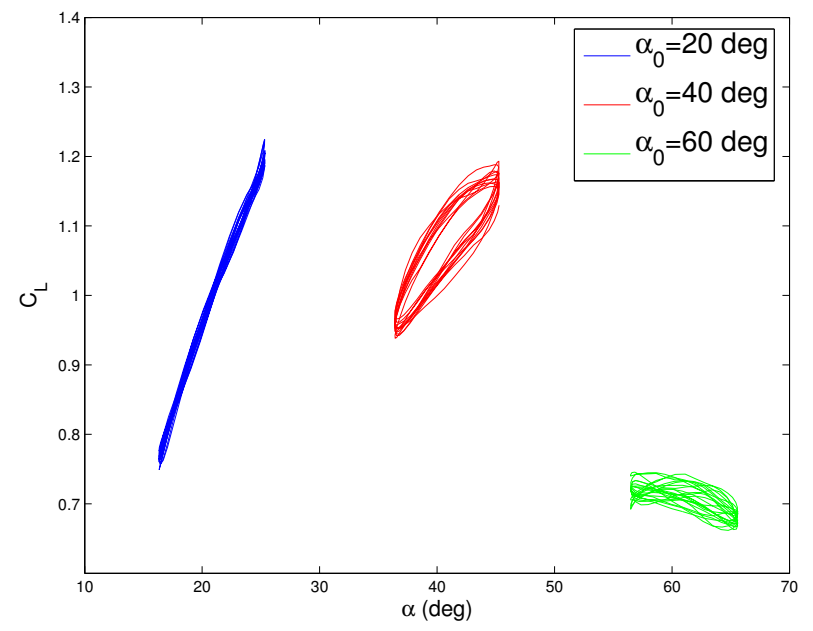

(b) $f=2.94 \mathrm{rad} / \mathrm{s}$

Figure 4. Small amplitude oscillatory $C_{L}$ data at different testing conditions and inputs

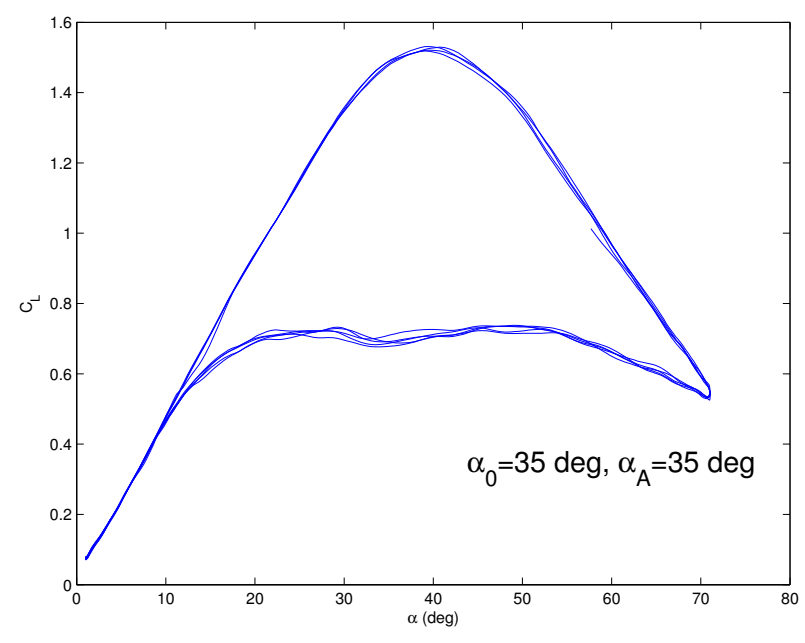

(a) $f=0.25 \mathrm{~Hz}, \alpha_{A}=35^{\circ}$

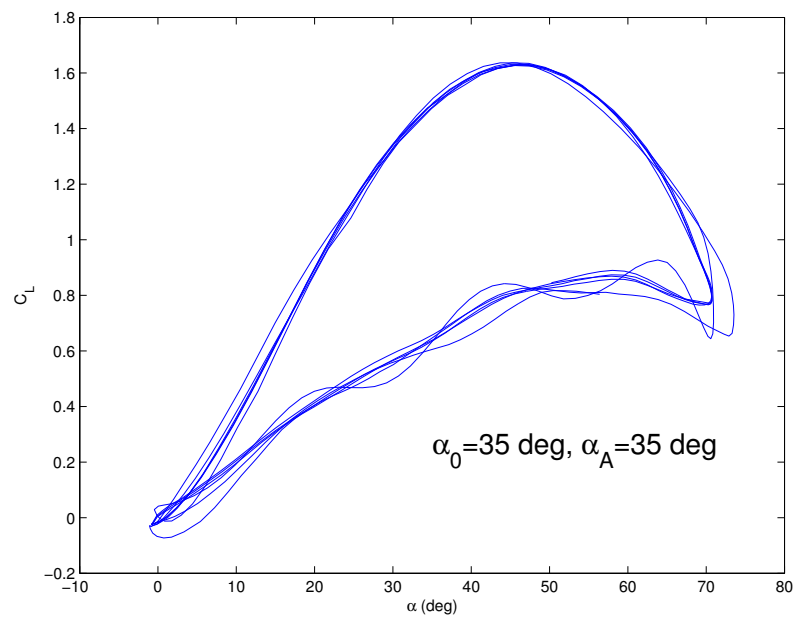

(b) $f=1 \mathrm{~Hz}, \alpha_{A}=35^{\circ}$

Figure 5. Large amplitude oscillatory $C_{L}$ data at different testing conditions and inputs 


\section{B. Model Structure Development}

For one degree of freedom motion in pitch, the fundamental relations for drag, lift and pitching moment have the form:

$$
C_{a}(t)=C_{a}(\alpha(t), q(t))
$$

where $a=D, L$ or $m$. Tobak and Schiff ${ }^{22}$ formulated these aerodynamic coefficients as

$$
C_{a}(t)=C_{a}(0)+\int_{0}^{t} C_{a_{\alpha}}(t-\tau ; \alpha(\tau), q(\tau)) \dot{\alpha}(\tau) d \tau+\frac{l}{V} \int_{0}^{t} C_{a_{q}}(t-\tau ; \alpha(\tau), q(\tau)) \dot{q}(\tau) d \tau
$$

where $C_{a}(0)$ is the initial condition and $C_{a_{\alpha}}$ and $C_{a_{q}}$ are indicial functions representing the change in aerodynamic coefficient due to (separately applied) unit steps in $\alpha$ and $q$. Following Klein and Noderer ${ }^{8}$ and Klein and Murphy ${ }^{10}$ we write these indicial functions as:

$$
\begin{gathered}
C_{a_{\alpha}}(t-\tau ; \alpha(\tau), q(\tau))=C_{a_{\alpha}}(\infty ; \alpha(\tau), q(\tau))-F_{a_{\alpha}}(t-\tau ; \alpha(\tau), q(\tau)) \\
C_{a_{q}}(t-\tau ; \alpha(\tau), q(\tau))=C_{a_{q}}(\infty ; \alpha(\tau), q(\tau))-F_{a_{q}}(t-\tau ; \alpha(\tau), q(\tau))
\end{gathered}
$$

where the deficiency functions $F_{a_{\alpha}}$ and $F_{a_{q}}$ tend to zero as $t-\tau$ tend to infinity. Substituting these equations into (14) and assuming that the initial condition $C_{a}(0)$ is zero, we obtain:

$$
C_{a}(t)=C_{a}(\infty ; \alpha(t), q(t))-\int_{0}^{t} F_{a_{\alpha}}(t-\tau ; \alpha(\tau), q(\tau)) \dot{\alpha}(\tau) d \tau-\frac{l}{V} \int_{0}^{t} F_{a_{q}}(t-\tau ; \alpha(\tau), q(\tau)) \dot{q}(\tau) d \tau
$$

where $C_{a}$ is the total (due to $\alpha$ and $q$ ) aerodynamic coefficient that corresponds to steady flow with both $\alpha$ and $q$ fixed at their respective instantaneous values. This is the indicial response function model of aerodynamic coefficients given by Klein and Murphy. ${ }^{10}$ Reference 10 goes on to suggest expressing the deficiency functions around $q=0$ as:

$$
F(t ; \alpha, 0)=\sum_{i=1}^{N} c_{i}(\alpha) e^{-a_{i} t}
$$

and expanding $C_{a}$ in Taylor's series around $q=0$. This leads us to the model structure for lift coefficient

$$
\begin{aligned}
C_{L}(t)= & C_{L}(\alpha(t))+\frac{l}{V} C_{L_{q}}(\alpha(t)) \dot{\alpha}(t)-\sum_{i=1}^{N} \int_{0}^{t} c_{\alpha i}(\alpha(\tau)) e^{-a_{\alpha i}(t-\tau)} \dot{\alpha}(\tau) d \tau \\
& -\frac{l}{V} \sum_{i=1}^{M} \int_{0}^{t} c_{q i}(\alpha(\tau)) e^{-a_{q i}(t-\tau)} \ddot{\alpha}(\tau) d \tau
\end{aligned}
$$

where we used the fact that, in one degree of freedom pitch motion, $q=\dot{\alpha}$. The model structures for drag and pitching moment are similar.

Define the state space matrices

$$
A=\text { diagonal }\left\{a_{\alpha 1}, \cdots, a_{\alpha N}, a_{q 1}, \cdots, a_{q M}\right\}, \quad B=\left[\begin{array}{cccccccc}
1 & 1 & \cdots & 1 & 0 & 0 & \cdots & 0 \\
0 & 0 & \cdots & 0 & 1 & 1 & \cdots & 1
\end{array}\right]^{T}
$$

12 of 18

American Institute of Aeronautics and Astronautics 


$$
C(\alpha)=-\left[c_{\alpha 1}(\alpha), \cdots, c_{\alpha N}(\alpha), c_{q 1}(\alpha), \cdots, c_{q M}(\alpha)\right]
$$

and note that (15) is equivalent to the structured state space model

$$
\begin{aligned}
& \dot{x}(t)=A x(t)+B\left[\begin{array}{c}
\dot{\alpha}(t) \\
\ddot{\alpha}(t)
\end{array}\right] \\
& y(t)=C(\alpha(t)) x \\
& C_{L}(t)=C_{L}(\alpha(t))+\frac{l}{V} C_{L_{q}}(\alpha(t)) \dot{\alpha}(t)+y(t)
\end{aligned}
$$

where the $A$-matrix is independent of $\alpha$. More generally, we may take the diagonal entries of $A$ to be functions of $\alpha$ to create an LPV system. With $N=1$ and $M=1$, such a model takes the form:

$$
\begin{aligned}
& \dot{x}(t)=\left[\begin{array}{cc}
a_{1}(\alpha(t)) & 0 \\
0 & a_{2}(\alpha(t))
\end{array}\right] x(t)+\left[\begin{array}{c}
\dot{\alpha}(t) \\
\ddot{\alpha}(t)
\end{array}\right] \\
& y(t)=\left[\begin{array}{ll}
c_{1}(\alpha(t)) & c_{2}(\alpha(t))
\end{array}\right] x \\
& C_{L}(t)=C_{L}(\alpha(t))+\frac{l}{V} C_{L_{q}}(\alpha(t)) \dot{\alpha}(t)+y(t)
\end{aligned}
$$

which will be used for identification. The $\alpha$-dependent terms $a_{1}, a_{2}, c_{1}, c_{2}, C_{L}(\alpha(t))$ and $C_{L q}(\alpha(t))$ are all assumed to be polynomials in $\alpha$.

\section{Identification Results}

Though it is possible to estimate all the model parameters simultaneously, we follow the sequential approach given below to estimate model parameters:

1. Determine polynomial coefficients of the static term $C_{L}(\alpha(t))$ using static test data.

2. Adjust the small amplitude oscillatory aerodynamic coefficient data by subtracting out the contribution from static term due to oscillatory $\alpha$ input:

$$
\widetilde{C}_{L}(t)=C_{L}(t)-C_{L}(\alpha(t))
$$

where $C_{L}(t)$ denotes the measured lift coefficient in response to small amplitude $\alpha$ input, and $C_{L}(\alpha(t))$ is the identified static term at the test input $\alpha(t)$.

3. Determine polynomial coefficients of the damping term $C_{L q}$ from the adjusted small amplitude test data

4. Adjust the large amplitude oscillatory aerodynamic coefficient data by subtracting out the contribution from static and damping terms due to oscillatory $\alpha$ input:

$$
\widetilde{C}_{L}(t)=C_{L}(t)-C_{L}(\alpha(t))-\frac{l}{V} C_{L_{q}}(\alpha(t)) \dot{\alpha}(t)
$$

where $C_{L}(t)$ denotes the measured lift coefficient in response to large amplitude $\alpha$ input.

13 of 18

American Institute of Aeronautics and Astronautics 
5. Identify a LPV state space model of the form (16a-16b) to best fit the adjusted large amplitude oscillatory data using the residue-based algorithm for LPV models.

We obtained:

$$
C_{L}(\alpha)=-0.004+1.72 \alpha+11.8 \alpha^{2}-32.2 \alpha^{3}+26.77 \alpha^{4}-7.4 \alpha^{5}
$$

from static data. The small amplitude oscillatory data corresponding to the frequency $f=1 \mathrm{~Hz}$ were adjusted for identifying $C_{L q}$. This resulted in

$$
\frac{l}{V} C_{L q}=-0.7+2.86 \alpha-2.05 \alpha^{2}
$$

Finally, the large amplitude data corresponding to $\alpha_{A}=35^{\circ}$ and $f=0.75,1 \mathrm{~Hz}$ were adjusted and used to identify the following LPV model:

$$
\begin{aligned}
& \dot{x}(t)=\left[\begin{array}{cc}
-9.7 & 0 \\
0 & -8.8
\end{array}\right] x(t)+\left[\begin{array}{ll}
1 & 0 \\
0 & 1
\end{array}\right]\left[\begin{array}{c}
\dot{\alpha}(t) \\
\ddot{\alpha}(t)
\end{array}\right] \\
& y(t)=\left[\begin{array}{ll}
c_{1}(\alpha(t)) & c_{2}(\alpha(t))
\end{array}\right] x
\end{aligned}
$$

where

$$
c_{1}(\alpha)=0.88+6.55 \alpha-5.12 \alpha^{2} \text { and } c_{2}(\alpha)=-2.03-1.07 \alpha+8.88 \alpha^{2}-5.53 \alpha^{3}
$$

The dependency of $A$ matrix on $\alpha$ was found to be small and neglected in (19a). The complete model for $C_{L}$ is given by (16c). Figures 6-7 show comparison of response of identified model with the data used for identification and with the large amplitude oscillatory data at $\alpha_{A}=20^{\circ}$.

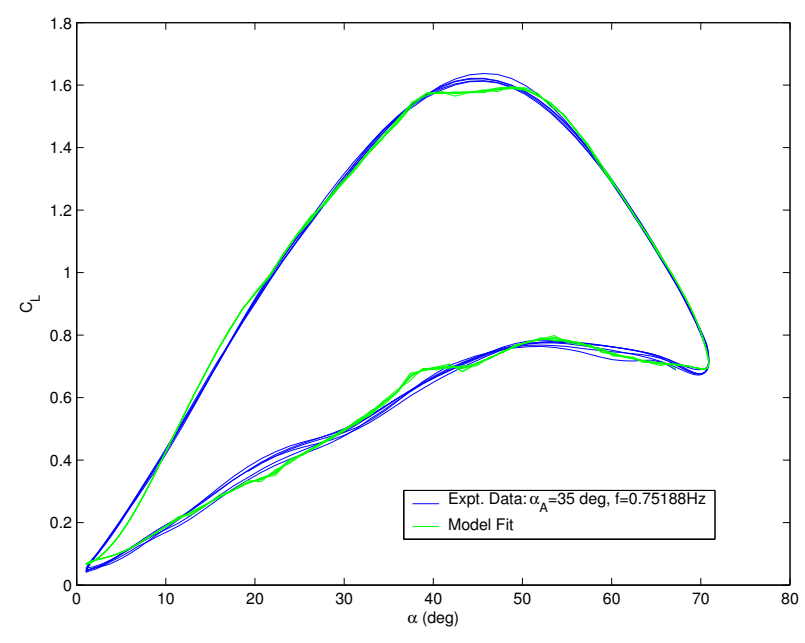

(a) $\alpha_{A}=35^{\circ}, f=0.75 \mathrm{~Hz}$

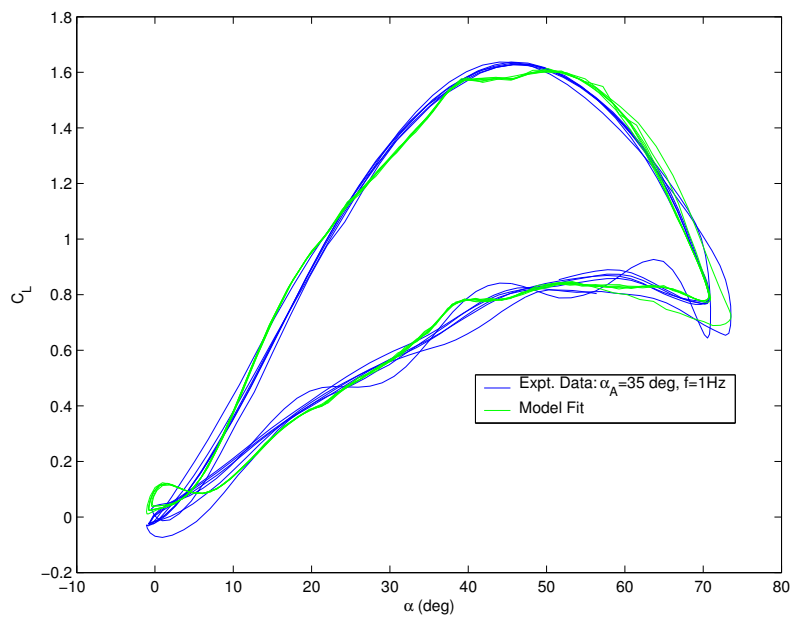

(b) $\alpha_{A}=35^{\circ}, f=1 H z$

Figure 6. Comparison of response of identified model and large amplitude test data

The ramp data was used to test the validity of the identified model. Figures 8-9 show comparisons of experimental data and output of identified model. These figures show good agreement up to a 


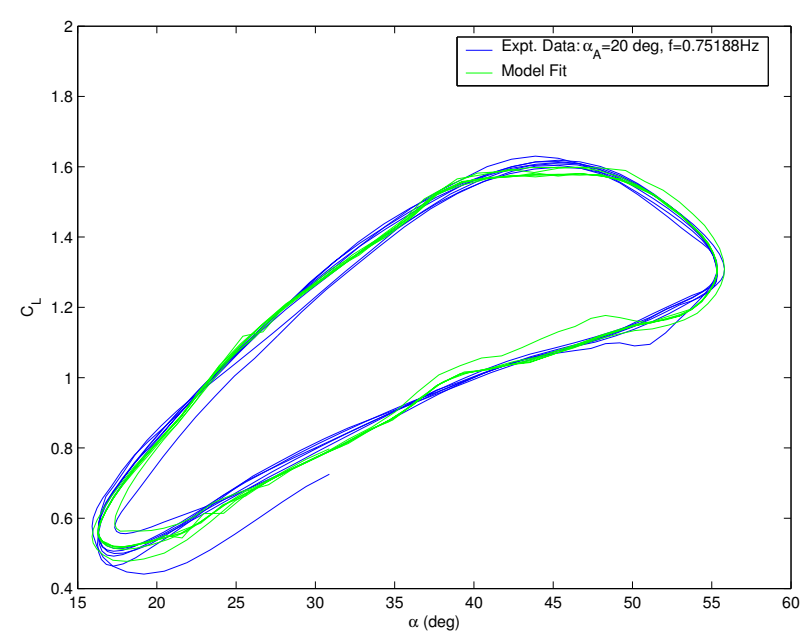

(a) $\alpha_{A}=20^{\circ}, f=0.75 H z$

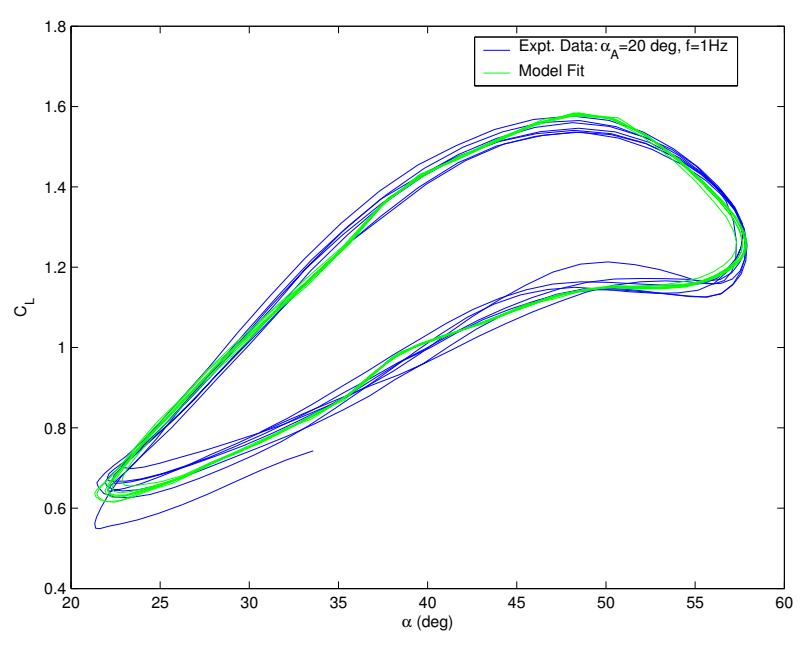

(b) $\alpha_{A}=20^{\circ}, f=1 \mathrm{~Hz}$

Figure 7. Comparison of predicted response of identified model and large amplitude test data

ramp rate of $18^{\circ} / \mathrm{s}$. A part of the error is due to initial conditions which where set to zero for the identified model. The largest percentage error occurred at a ramp rate of $18^{\circ} / \mathrm{s}$ as shown on the right in Figure 9.

\section{Conclusions and Directions for Future Research}

This paper presented local and parameter dependent model structures for modeling unsteady aerodynamic effects. We have also developed additional nonlinear model structures details of which can be obtained from the authors. Table 1 gives a summary of the model structures. The unstructured model identification can be carried out using the subspace method and its extensions to the nonlinear case. The structured model identification can be carried out using the residue algorithm and its extensions.

The algorithms were evaluated on a limited set of wind tunnel data for the F-16XL provided by NASA Langley Research Center. Our experience has been that the model structures and algorithms are well suited for modeling unsteady and nonlinear aerodynamic effects, in particular the structured models. It should be noted that the key issue of model structure determination is still un-resolved. We have no systematic procedure to determine model structures (other than trying out all the structures in a library of model structures). This issue is likely to remain difficult. The identification results presented in this report dealt with an aircraft with one lifting surface. This approach should be extended to a wing-tail configuration (some work along these lines can be found in Reference 11). In addition, modeling should be extended to aircraft non-planar motion taking into account the effect of angle-of-attack and side-slip on parameters. One of the main issues, 


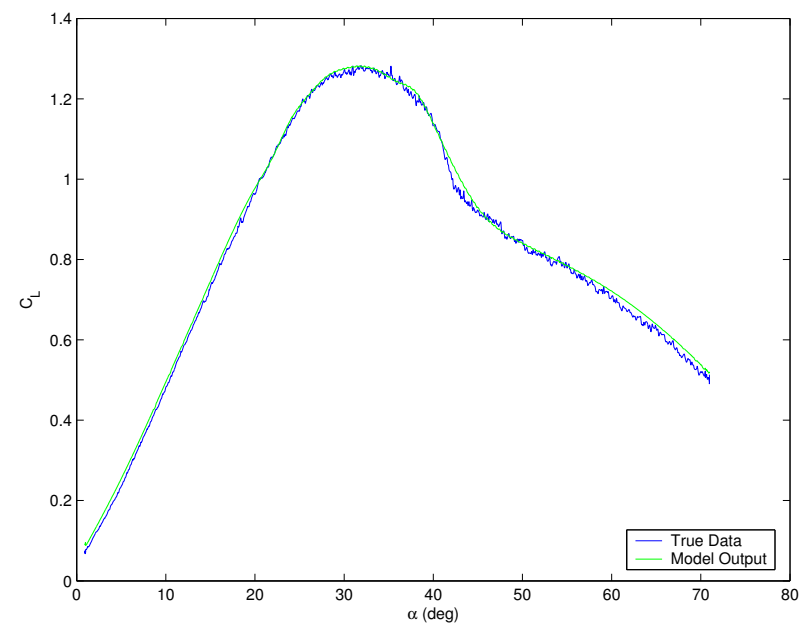

(a) Ramp up from $\alpha_{0}=0^{\circ}$ at rate $\dot{\alpha}=1^{\circ} / \mathrm{s}$

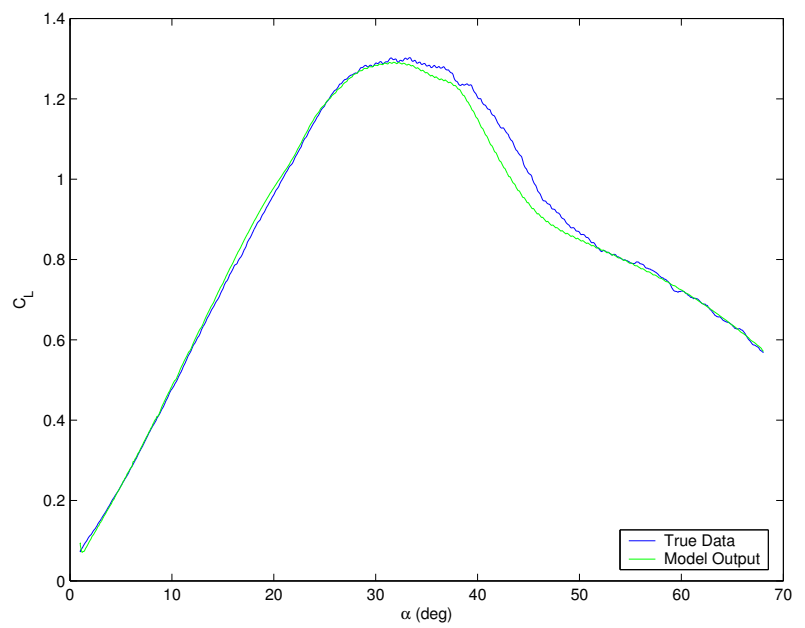

(b) Ramp up from $\alpha_{0}=0^{\circ}$ at rate $\dot{\alpha}=5^{\circ} / \mathrm{s}$

Figure 8. Comparison of predicted response of identified model and validation ramp test data

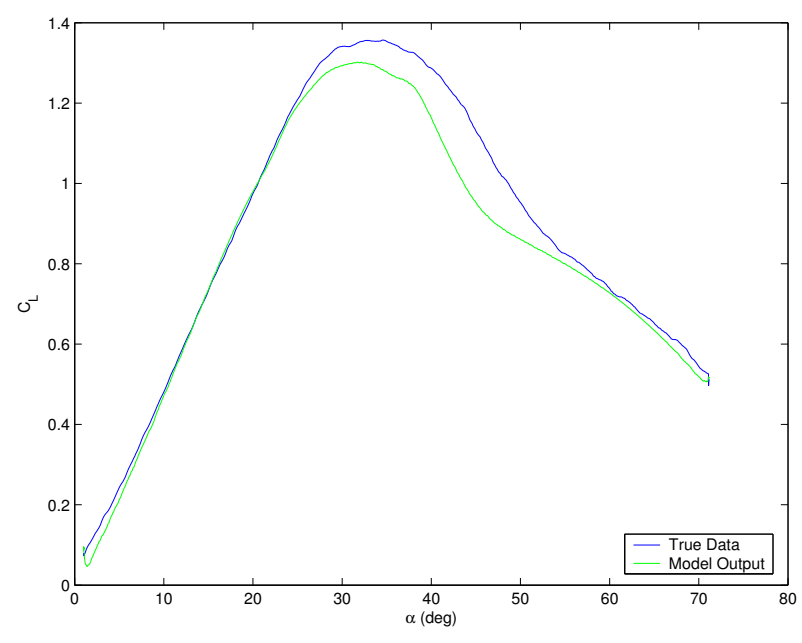

(a) Ramp up from $\alpha_{0}=0^{\circ}$ at rate $\dot{\alpha}=10^{\circ} / \mathrm{s}$

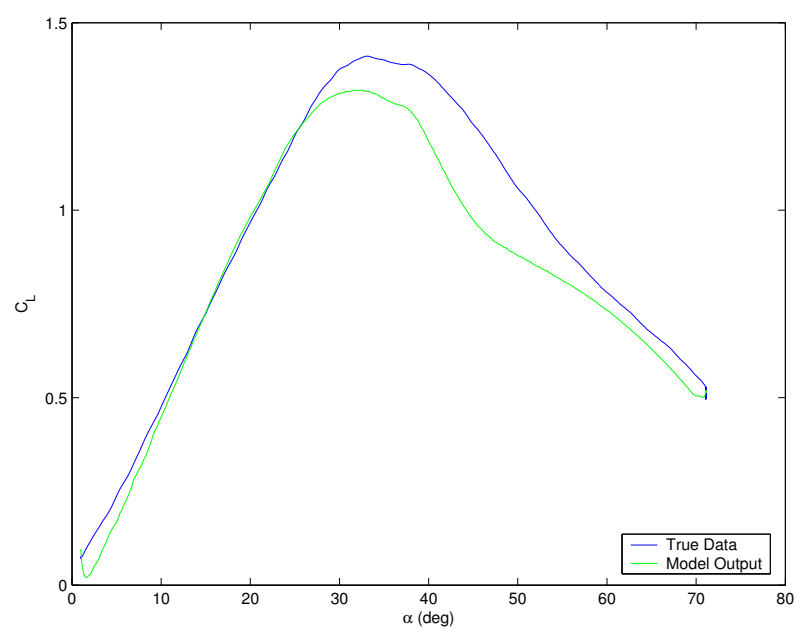

(b) Ramp up from $\alpha_{0}=0^{\circ}$ at rate $\dot{\alpha}=18^{\circ} / \mathrm{s}$

Figure 9. Comparison of predicted response of identified model and validation ramp test data 


\begin{tabular}{||c|c|c|c|c|c||}
\hline \hline Property & $\begin{array}{c}\text { Unstructured } \\
\text { LTI }\end{array}$ & $\begin{array}{c}\text { Structured } \\
\text { LTI }\end{array}$ & $\begin{array}{c}\text { Hammerstein } \\
\text { and Wiener }\end{array}$ & LPV & $\begin{array}{c}\text { Parameter-Dependent } \\
\text { Hammerstein/Wiener }\end{array}$ \\
\hline Linear & Yes & Yes & No & Yes & No \\
\hline Global & No & No & No & Yes & Yes \\
\hline $\begin{array}{c}\text { Apriori } \\
\text { Information }\end{array}$ & No & Yes & Yes & Yes & Yes \\
\hline Algorithm & Subspace & SID & Modified SID & Modified SID & Modified SID \\
\hline \hline
\end{tabular}

Table 1. Summary of Model Structures (SID-Structured Identification Algorithm)

as with the problems studied in this report, lies in model structure determination and analytical studies to gain a physical understanding of the flow effects should be undertaken. We suggest three additional areas for future research.

Identification of Reduced-order Models of CFD Solvers: Unsteady CFD solvers make the fewest assumptions about the flow and, in many cases, they can accurately predict flow around geometries. But, their inclusion in aircraft analysis and design is not feasible due to the high computational cost. The development of computationally efficient reduced order models of unsteady flow is thus of paramount importance. Though the focus of our work has been modeling of aerodynamic effects for flight dynamics, the models and algorithms described in this paper can be applied to obtain reduced order models of CFD solvers. It has potential applications in aeroservoelasticity and control of morphing wings.

Uncertainty Description: Nonlinear phenomenon such as Hopf bifurcations are properties of mathematical models that occur at specific parameter values. If these properties persist with respect to uncertainties, then we can be reasonably confident that the true system will also exhibit such properties. So, we suggest that useful models be required to supply a description of the uncertainties so that phenomena observed during analysis of an identified model can classified as artifacts of the model or physical phenomena. Computation of modeling uncertainties from data is a difficult problem. The maximum likelihood estimation (MLE) and Bayesian parameter estimation techniques may be able to determine parametric uncertainties. A deeper look into this issue is suggested for future work.

Experiment Design: The reliability of any model identified from experimental data is dependent to a great extent on the experiment itself. The problem of input design, also known as experiment design, has been the subject of vigorous research. At present, experiment design for linear system identification is well understood and there is a general framework for addressing constrained and nonlinear systems. See for instance the references 7,14,18,19. However, experiment design for high angle of attack flight dynamics and unstable flight regimes has not received the same level of attention. The use of experiment design methods in industry can reduce the amount of testing and improve safety.

17 of 18

American Institute of Aeronautics and Astronautics 


\section{References}

${ }^{1}$ Special section on flight vehicle system identification, Parts I and II, AIAA Journal of Aircraft, Vol. 41, Number 4 July-August/Vol. 42, Number 1, January-February, 2005.

${ }^{2}$ Invited session on identification of unsteady aerodynamic models for aircraft applications, AIAA Atmospheric Flight Mechanics Conference, August 16-19, Providence, 2004.

${ }^{3}$ H. Akaike, "A new look at the statistical model identification", IEEE Transactions on Automatic Control, Vol.19, No.6, December, 1974, pp.716-723.

${ }^{4}$ A. Dempster, N. Laird and D. Rubin, "Maximum likelihood from incomplete data via the EM algorithm", Journal of the Royal Statistical Society, Vol.39, No.1, Series B, 1977.

${ }^{5} \mathrm{M}$. Goman and A. Khrabrov, "State space representation of aerodynamic characteristics of an aircraft at high angles of attack", AIAA Paper 92-4651, 1992.

${ }^{6} \mathrm{M}$. Goman, A. Khrabrov and S. Usoltsev, "Identification of unsteady aerodynamic motion of a delta wing at high angles of attack", SYSID'94 Paper, 1994.

${ }^{7}$ G. C. Goodwin and R. Payne, Dynamic system identification: experiment design and data analysis, Academic Press, 1977.

${ }^{8}$ V. Klein and K. Noderer, "Modeling of aircraft unsteady aerodynamic characteristics - Part I,II,II", NASA TM109120, NASA TM-110161, NASA TM-110259, 1994-1996.

${ }^{9}$ V. Klein, P. Murphy, T. Curry and J. Brandon, "Analysis of Wind Tunnel Longitudinal Static and Oscillatory Data of the F-16XL Aircraft", NASA/TM-97-206276, December, 1997.

${ }^{10} \mathrm{~V}$. Klein and P. Murphy, "Estimation of aircraft nonlinear unsteady parameters from wind tunnel data. NASA TM-1998-208969, 1998.

${ }^{11}$ V. Klein, "Modeling of Longitudinal Unsteady Aerodynamics of a Wing-Tail Combination", NASA-CR-1999209547, September, 1999.

${ }^{12}$ S. Kim, P. Murphy and V. Klein, "Evaluation and Analysis of F-16XL Wind Tunnel Data from Static and Dynamic Tests", NASA/TM-2004-213234, June, 2004.

${ }^{13}$ L. Ljung, System Identification: Theory for the user, Second Edition, Prentice Hall, New York, 1999.

${ }^{14}$ R. K. Mehra, "Optimal input signals for parameter estimation in dynamic system: Survey and new results", IEEE Transactions on Automatic Control, Vol.19, No.6, December, 1974, pp.753-768.

${ }^{15}$ N. K. Gupta and R. K. Mehra, "Computational aspects of maximum likelihood estimation and reduction in sensitivity function calculations", IEEE Transactions on Automatic Control, Vol.19, No.6, December, 1974.

${ }^{16}$ R. K. Mehra, "A New Unified Approach to State Space Model Structure Determination and Maximum Likelihood Identification with Aerospace Applications," IFAC Symp. on System Identification, Copenhagen, July 1994.

${ }^{17}$ E. Morelli, "Global Nonlinear Parametric Modeling With Application To F-16 Aerodynamics", Proc. of American Control Conf., Philadelphia, 1998.

${ }^{18}$ E. Morelli, "Advances In Experiment Design For High Performance Aircraft", AGARD Symposium on System Identification for Integrated Aircraft Development and Flight Testing, Madrid, Spain, Paper No. 8, May 5-8, 1998.

${ }^{19}$ E. Morelli, "In-Flight System Identification", AIAA 98-4261, Proc. of AIAA Atmospheric Flight Mechanics Conference, Boston, 1998.

${ }^{20}$ E. Morelli, "SIDPAC: System IDentification Programs for Aircraft", AIAA 2002-4704, Proc. of AIAA Atmospheric Flight Mechanics Conference, Monterey, California, August 5-8, 2002.

${ }^{21}$ P. Murphy and V. Klein, "Estimation of Aircraft Unsteady Aerodynamic Parameters From Dynamic Wind Tunnel Testing", Proc. of AIAA Atmospheric Flight Mechanics Conference, Montreal, Canada, AIAA 2001-4016, August 6-9, 2001.

${ }^{22}$ M. Tobak and L. Schiff, "On the formulation of aerodynamic characteristics in aircraft dynamics", NASA TR-R-456, 1976.

${ }^{23}$ P. Van Overschee and B. De Moor, Subspace identification for linear systems, Kluwer, 1996.

18 of 18

American Institute of Aeronautics and Astronautics 